\title{
On the Concentration Dependence of GPC Elution Volumes
}

\author{
Josef JANČA \\ Institute of Macromolecular Chemistry, Czechoslovak Academy of Sciences, \\ 16206 Prague 6, Czechoslovakia.
}

(Received December 7, 1979)

KEY WORDS Concentration Effects / Elution Volume / GPC /

In a paper published recently, Mahabadi and Rudin $^{1}$ studied theoretically the concentration dependence of hydrolynamic volumes and elution volumes in gel-permeation chromatography (GPC). They compared two different theoretical models, describing the dependence of the effective dimensions of the macromolecular coil in solution (e.g., of the hydrodynamic volume) on the concentration. One of these models represents the earlier Yamakawa's theory, ${ }^{2}$ and the other, the recent, very flexible theory suggested by Rudin and Wagner. ${ }^{3}$ These authors ${ }^{1}$ also examined the suitability of their theory for the elucidation of the dependence of elution volumes in GPC on the concentration of injected polymer solutions. It is well-known that elution volumes in GPC increase with increasing concentration and this is sometimes called the concentration effect. A change in the effective size of macromolecules in solution caused by a change in concentration is doubtless operative in GPC, and consequently affects the elution volumes. Rudin and Hoegy ${ }^{4}$ documented the suitability of Rudin's original theory ${ }^{5}$ for GPC, but they did not consider concentration changes which occur during the chromatographic process due to dilution along the column. Although Mahabadi and Rudin ${ }^{1}$ stressed the fact that the suggested application of their theory to concrete GPC conditions is "valid only for sharp fractions for which the GPC separation process does not cause a significant dilution," they did not take into account the fact that monodisperse fractions are also diluted during their movement in the chromatographic column, not by the separation process itself, but by axial dispersion, and also, immediately at the column inlet by a distribution between the solvent inside and outside the pores. Quite obviously, the higher the efficiency of the chromatographic system, the smaller the dilution of the solution in the chromatographic zone. However, even in columns with high efficiency, significant dilution occurs, so that the concentration at the column outlet is several times lower than that of the injected solution.

Mahabadi and Rudin ${ }^{1}$ used the experimental results from our paper ${ }^{5}$ dealing with concentration effects in GPC, in order to demonstrate the quantitative agreement between the theoretically calculated dependence of the elution volume on concentration and experimental data. In a graphic plot which shows this aparent agreement by using our experimental data, ${ }^{6}$ they employed concentrations of the polystyrene standard solutions at the moment of injection ( $c f$. Figure 6b, in ref 1). As has been pointed out above all processes occurring in the chromatographic column and causing cont'muous dilution of the polymer solution in the zone moving along the column should be taken into account. To obtain a graphic plot such as that in Figure $6 \mathrm{~b}$, in ref 1 , it is necessary to calculate the average effective concentrations, e.g., using the relation given in our original paper ${ }^{6}$ or in another paper of this series. ${ }^{7-13}$ In our recent papers, ${ }^{7-13}$ we employed the revised Rudin's theory ${ }^{3}$ and also corrected some quantitative conclusions of our original paper ${ }^{6}$ with respect to the deformed shape of the elution curves of polymers at higher concentrations, which had to be described in terms of statistical moments. The basic principle of our theoretical conception of concentration effects in GPC is that several participating processes contri- 
bute to the resulting change in the elution volumes with varying concentration. One of these processes, but not the predominate one, also involves a change in the effective dimensions of the macromolecules in solution. This change in the hydrodynamic volume causes a change in the elution volume or in the distribution coefficient according to the respective calibration curve. Both our theoretical calculations and the experimental results ${ }^{6-13}$ show that the prevailing factor which contributes to the concentration effects consists in viscosity phenomena in the interstitial volume, and thus in the dynamic processes in the mobile phase, which, under our experimental conditions, amounted to some 80 $90 \%$ of the total change in the elution volumes with varying concentration.

If we plot the dependence of the elution volume on concentration as did Mahabadi and Rudin ${ }^{1}$ ( $c f$. Figure $6 \mathrm{~b}$ of the reference cited above), by recalculating the experimental points of our original paper $^{6}$ (so as to take into account zone spreading along the column) and by using the average concentration instead of the solution concentration at the moment of injection, we obtain the results shown in Figure 1. The average concentration $c_{\text {ave }}$ along the column was calculated using the following relations ${ }^{6,9,11}$ :

$$
c_{\text {ave }}=\int_{0}^{L} c_{x} \mathrm{~d} x / \int_{0}^{L} \mathrm{~d} x
$$

where $c_{\mathrm{x}}$ is an arbitrary concentration (e.g., in the range of the maximum of the concentration profile on the column) in the column coordinate $x$, and $L$ is the column length. It also holds ${ }^{6,11}$ that

$$
c_{\mathrm{x}}=\delta_{\mathrm{I}} c_{\mathrm{I}} / \delta_{\mathrm{x}}
$$

and

$$
\delta_{\mathrm{x}}^{2}=\delta_{\mathrm{I}}^{2}+x\left(\delta_{\mathrm{T}}^{2}-\delta_{\mathrm{I}}^{2}\right) / L
$$

where $c_{1}$ is the concentration of the injected-polymer solution, $\delta_{\mathrm{I}}$ is the standard deviation of the injection function, which may be approximated by the Gaussian function, and $\delta_{\mathrm{T}}$ is the standard deviation of the elution curve (with neglected end effect ${ }^{14}$ ). By solving eq 1 and using eq 2 and 3, we obtain

$$
c_{\mathrm{ave}}=2 \delta_{\mathrm{I}} c_{\mathrm{I}} /\left(\delta_{\mathrm{T}}+\delta_{\mathrm{I}}\right)
$$

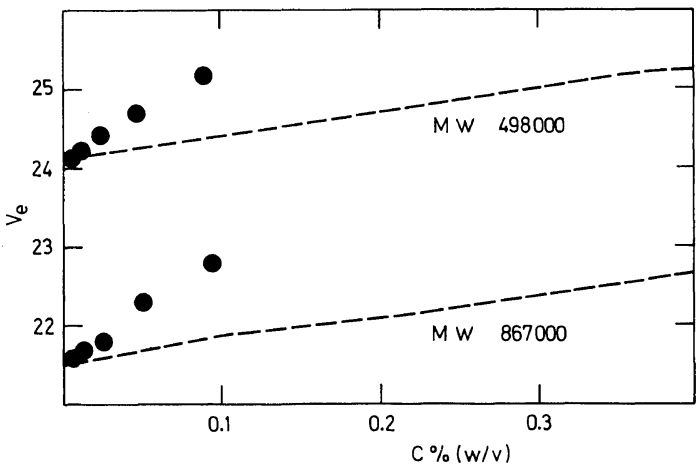

Figure 1. Concentration dependence of the elution volume of two different polystyrene standards: ---, calculated curves, ref $1 ; 0$, experimental points, ref 6 .

Figure 1 clearly shows that the experimentally determined dependence of the elution volume on concentration is much steeper than corresponds to the theoretical calculation, ${ }^{1}$ and thus the effect of the change in the hydrodynamic volume with changing concentration is a process that only partly contributes to the overall concentration effect. The values $\delta_{\mathrm{I}}, \delta_{\mathrm{T}}$, and $c_{\mathrm{I}}$ needed for calculating $c_{\text {ave }}$ were taken from our original paper. ${ }^{6}$

\section{REFERENCES}

1. H. K. Mahabadi and A. Rudin, Polym. J., 11, 123 (1979).

2. H. Yamakawa, J. Chem. Phys., 43, 1334 (1965).

3. A. Rudin and R. A. Wagner, J. Appl. Polym. Sci., 20, 1483 (1976).

4. A. Rudin and H. L. W. Hoegy, J. Polym. Sci., A-1, 10, 217 (1972).

5. A. Rudin, J. Polym. Sci., A-1, 9, 2587 (1971).

6. J. Janča, J. Chromatogr., 134, 263 (1977).

7. J. Janča and S. Pokorný, J. Chromatogr., 148, 31 (1978).

8. J. Janča and S. Pokorný, J. Chromatogr., 156, 27 (1978).

9. J. Janča, J. Chromatogr., 170, 309 (1979).

10. J. Janča and S. Pokorný, J. Chromatogr., 170, 319 (1979).

11. J. Janča, Anal. Chem., 51, 637 (1979).

12. J. Janča, J. Chromatogr., in press.

13. J. Janča, S. Pokorný, M. Bleha, and O. Chiantore, $J$. Liq. Chromatogr., submitted.

14. J. Janča, J. Liq. Chromatogr., 1, 731 (1978). 